\title{
Harmonic Cut and Regularized Centroid Transform for Localization of Subcellular Structures
}

\author{
Qing Yang, Member, IEEE, and Bahram Parvin, Senior Member, IEEE
}

\begin{abstract}
Two novel computational techniques, harmonic cut and regularized centroid transform, are developed for segmentation of cells and their corresponding substructures observed with an epi-fluorescence microscope. Harmonic cut detects small regions that correspond to small subcellular structures. These regions also affect the accuracy of the overall segmentation. They are detected, removed, and interpolated to ensure continuity within each region. We show that interpolation within each region (subcellular compartment) is equivalent to solving the Laplace equation on a multi-connected domain with irregular boundaries. The second technique, referred to as the regularized centroid transform, aims to separate touching compartments. This is achieved by adopting a quadratic model for the shape of the object and relaxing it for final segmentation.
\end{abstract}

Index Terms-cell segmentation, regularization, interpolation, harmonic function, speckle noise

\section{INTRODUCTION}

Automatic segmentation of cells and their compartments is an important step for high-throughput quantitative biology. The biology of cellular responses, protein expression, and morphological structure is inherently heterogeneous due to cell cycle and environmental factors. If segmentation can be automated, then it provides the foundation for quantitative population studies for protein expression and model construction at subcellular scale [1], [2]. Yet, reliable segmentation is difficult since images are noisy, cellular stain is heterogeneous, and various compartments could overlap during the imaging process. This paper focuses on defining analytical and computational operators for segmenting cells and their compartments observed through an epi-fluorescence microscope. Proposed technique is model-based and constructs on known geometric shape properties to avoid ad-hoc protocols based on thresholding and morphological operators. Figure 1 shows two examples where staining is non-uniform, images are corrupted as a result of random (CCD noise) and speckle noise (internal substructures), and neighboring compartments can potentially overlap. These images are obtained through confocal and wide field microscopy respectively. Thresholding is often unreliable for images obtained through wide field microscopy since the intensity distribution lacks uniformity. Figure 1 indicates that direct thresholding (even manual) [3] and edge detection techniques are not sufficient for delineating these cells over hundreds of images. The central issue is whether an analytical

Manuscript received 24 Apr. 2002; revised 27 Sep. 2002; accepted 29 Nov. 2002.

Recommended for acceptance by A. Campilho.

The authors are with the Imaging and Informatics Group of the Computational Science Division at Lawrence Berkeley National Laboratory, Berkeley, CA 94720 foundation can be developed to remove speckle noise and separate overlapping compartments. Although our results are limited to 2D images, e.g., a fixed focal plane, these techniques are also applicable to segmentation of 3D data sets as well.

The proposed model-based approach is shown in Figure 2, which aims to decouple a specific imaging artifact at each computational step. This model assumes that (1) speckle noise (often corresponding to chromatin in nuclei or mitochandrial in cytoplasm) can be extracted with elliptic features, and (2) the boundary of a nucleus is locally quadratic, which is true for almost all cells. Once speckle noise is detected, it is interpolated using harmonic cut without introducing artifacts. Next, touching nuclei are separated by collapsing each nucleus to its local centroid through a process called regularized centroid transform. The net result is a vector field, which can be easily partitioned. The layered protocol demonstrates our approach for constructing an analytical model, based on scale and geometric constraints, for delineating subcellular compartments. The approach is automated and is routinely used in our production environment [4].

The organization of this paper is as follows. Section II summarizes previous research. Section III outlines the details for detecting elliptic features. Section IV outlines details of harmonic cut and its solution with the Laplacian. Sections VVII present the regularized centroid transform and its implementation. Section VIII concludes the paper.

\section{Previous Research}

Previous research in cell segmentation has included both 2D data from wide field microscopy and 3D data from confocal or deconvolution microscopy. These techniques include Hough transform [5], perceptual grouping of step and roof edges [6], level set approach [7], watershed-based techniques [8], and iterative thresholding [2]. There are also a number of techniques that are based on minimizing a cost function using local geometrical features [9]-[11]. The Hough transform has the advantage of a parameterized model, but often generates many false primitives or multiple shape inferences (at the same location) due to variation in scale and edge intensity. Although perceptual organization of step and roof edges has a strong foundation, it suffers from lack of reliability that is associated with limited geometric constraints. In this system, step edges correspond to the boundaries between cells and background, while the roof edges correspond to boundaries between cells. The level set approach, as applied to the 3D data stacks, is interactive and has a limited application for high-throughput analysis. Furthermore, special consideration 


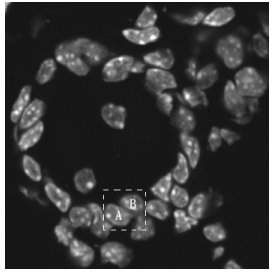

(a)

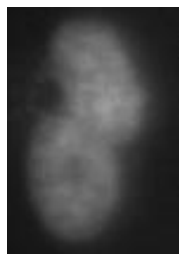

(d)

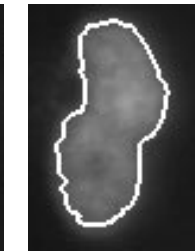

(e)

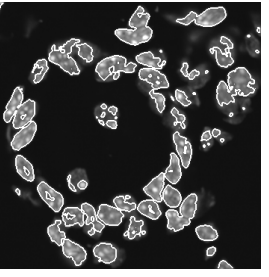

(b)

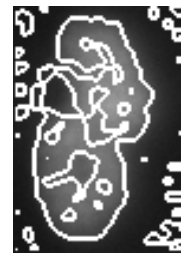

(f)

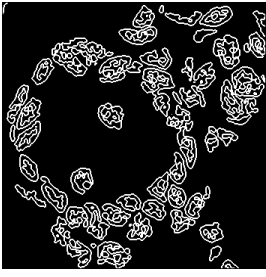

(c)
Fig. 1. Direct thresholding or detection of zero-crossings are not adequate for correct segmentation of a fixed tissue: (a) an original image observed with a confocal microscope; (b) contours corresponding to thresholded results by careful interactive selection of a threshold value of 90 between nuclei A and $\mathrm{B}$; (c) zero crossing result with sigma of 1.5 ; (d) original image from a widefield microscope of two touching nuclei of living cells where there is close to $50 \%$ variation in intensity between the edge of the cell and its centroid; (e) contours corresponding to thresholded results at 80; (f) zero crossing result with sigma of 2.0.

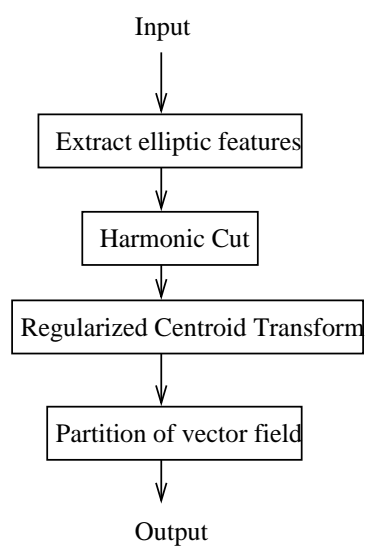

Fig. 2. Computational steps in noise removal and delineation of touching subcompartments.

must be made to filter out internal substructures during the evolution process. Still, the end result is only a representation and not a description, and edges have to be inferred in the post-processing step. The watershed techniques construct an energy map in the gradient direction based on the distance transform. This approach often leads to over-segmentation, which requires complex region merging protocol at a later stage. The over-segmentation is due to noise in the distance transform in the absence of regularization.

Our approach is model-based and well constrained to detect and localize subcellular compartments. The model characterizes the speckle noise as aggregates of a few pixels standing sharply above or below their immediate background, which can be detected, extracted, and interpolated. It then separates touching compartments by assuming a quadratic model of boundary. Our approach is automated to allow for highthroughput analysis, and it has been tested over a large data set.

\section{ELLIPTIC REGIONS}

The first step of the computational process is to detect and remove noise. In this case, the main concern is the speckle noise, which are small regions (in the order of several pixels) that stand significantly above or below their immediate background. These regions are detected with elliptic features, and then interpolated in the subsequent step. Let the linear scale-space representation of the original image $I_{0}(x, y)$ at scale $\sigma$ be given by $I(x, y ; \sigma)=I_{0}(x, y) * G(x, y ; \sigma)$ where $G(x, y ; \sigma)$ is the Gaussian kernel. For simplicity $I(x, y ; \sigma)$ is also denoted as $I(x, y)$ below. At each point $(x, y)$, the isointensity contour is defined by

$$
I(x+\Delta x, y+\Delta y)=I(x, y)
$$

where $(\Delta x, \Delta y)$ is the displacement vector. Expanding and truncating the above equation using Taylor's series, we have the following estimation:

$$
\frac{1}{2}(\Delta x, \Delta y) H(x, y)(\Delta x, \Delta y)^{T}+\left(I_{x}, I_{y}\right)(\Delta x, \Delta y)^{T}=0
$$

where

$$
H(x, y)=\left(\begin{array}{ll}
I_{x x} & I_{x y} \\
I_{x y} & I_{y y}
\end{array}\right)
$$

is the Hessian matrix of $I(x, y)$. The entire image domain is divided by Equation 1 into two parts:

$$
I(x+\Delta x, y+\Delta y)>I(x, y)
$$

and

$$
I(x+\Delta x, y+\Delta y)<I(x, y)
$$

or locally

$$
\frac{1}{2}(\Delta x, \Delta y) H(x, y)(\Delta x, \Delta y)^{T}+\left(I_{x}, I_{y}\right)(\Delta x, \Delta y)^{T}>0
$$

and

$$
\frac{1}{2}(\Delta x, \Delta y) H(x, y, z)(\Delta x, \Delta y)^{T}+\left(I_{x}, I_{y}\right)(\Delta x, \Delta y)^{T}<0
$$

If $H(x, y)$ is positive definite, then the region defined by Equation 3 is locally convex. Similarly, if $H(x, y)$ is negative definite, then the region defined by Equation 4 is locally convex. To determine whether $H(x, y)>0$ or $H(x, y)<0$, we analyze this feature in both cases:

1) $H(x, y)>0$. Then we have $I_{x x}>0, I_{y y}>0$, and hence $I_{x x}+I_{y y}>0$, and positive Laplacian means that $(x, y)$ is a "dark point", i.e., a point that is darker than its neighbors.

2) $H(x, y)<0$. Then we have $I_{x x}<0, I_{y y}<0$, and hence $I_{x x}+I_{y y}<0$, and negative Laplacian means that $(x, y)$ is a "bright point", i.e., a point that is brighter than its neighbors. 
Classifying elliptic points as bright or dark points is an important initial step of our computational process, since it provides a natural way to reduce information content by partitioning objects of interest. Furthermore, these regions have direct biological interpretations. For example, tiny bright regions (a few pixels across) within the nuclei often correspond to chromatin (see Figure 1a), and similar dark regions within the nuclei correspond to nucleoli (see Figure 1d). From a computational perspective, we have the following definition.

Definition 1: A point is a bright (dark) elliptic feature at scale $\sigma$ if the Hessian matrix of $I(x, y ; \sigma)$ is negative (positive) definite at this point.

In scale-space theory [12], $I_{x x} I_{y y}-I_{x y}^{2}$ is referred to as the elliptic feature, which corresponds to the basic form of partial differential equation. Figure 3 illustrates detection of elliptic features under ideal conditions such as uniform intensity with added white noise. Furthermore, we show that even in the presence of fragmentation, elliptic features can be grouped into convex sets to represent higher levels of representation. The first row shows the raw data with and without random noise ((b) and (d) are obtained by adding white noise to (a) and (c), respectively). The second row shows that the bright elliptic features for each of the images is incomplete at a given scale and can be highly fragmented. A potential highlevel abstraction may include grouping of fragmented regions for convexity and enclosure, as shown in the third row. This technique, however, fails in most $2 \mathrm{D}$ images due to variations in cell sizes and staining, irregular shapes, and touching cells.

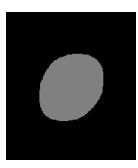

(a)

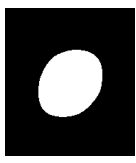

(e)

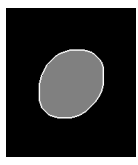

(i)

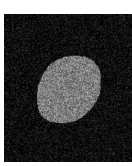

(b)

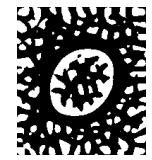

(f)

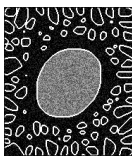

(j)

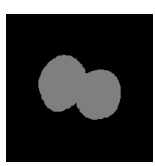

(c)

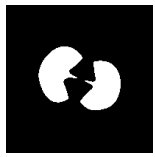

(g)

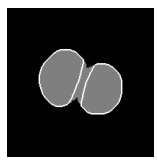

(k)

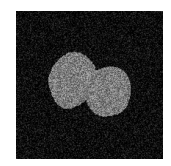

(d)

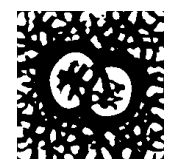

(h)

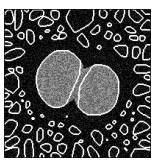

(1)
Fig. 3. Detection and grouping of elliptic features: (row 1) original synthetic images without (a and c) and with (b and d) added white noise; (row 2) detected bright elliptic features; and (row 3) grouping based on convexity and enclosure.

\section{HARMONIC CUT}

The next step of the computational process is to remove small elliptic regions, corresponding to small substructures

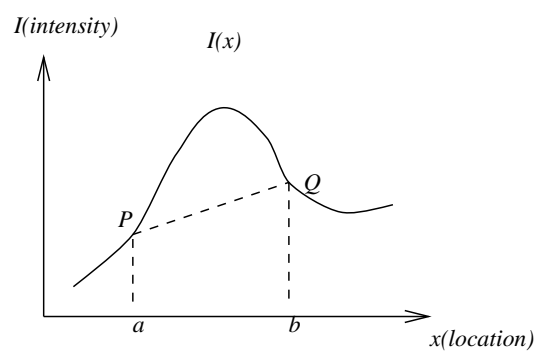

Fig. 4. 1-dimensional harmonic cut

within the nuclei, and interpolate these regions. This step corresponds to removing noise, which may be either random noise (CCD noise) or more importantly speckle noise (internal structures within the cell). To motivate our solution, consider the one-dimensional interpolation problem of Figure 4 with function $I(x)$ in the interval of $(a, b)$. The simplest method is to fill this interval with the average of the two endpoints, $\frac{I(a)+I(b)}{2}$, or with the value of the closest endpoint at every point on $(a, b)$, but these techniques break continuity or smoothness of interpolation. A better approach is to weight the interpolation, at each point $x$, as a function of its distance to the boundary condition, e.g., let $I^{n e w}(x)=[(b-x) I(a)+$ $(x-a) I(b)] /(b-a)$. This linear interpolation is as if we cut the function from $P$ to $Q$. The new function is continuous with vanishing second derivative on $(a, b)$. It is easy to show that this representation is equivalent to minimizing $\frac{1}{2} \int_{a}^{b} I_{x}^{2} d x$ subject to the boundary conditions $\left\{\begin{array}{l}I(a)=I_{a} \\ I(b)=I_{b}\end{array}\right.$

The $2 \mathrm{D}$ case is more complex because the boundary of the region to be removed is often noisy and irregular, and it is not clear whether propagating intensity based on distance transform will have desirable properties. One way to ensure continuity is to regularize the solution by extending the 1D solution to $2 \mathrm{D}$ or by minimizing the following functional:

$$
\frac{1}{2} \iint_{D} I_{x}^{2}+I_{y}^{2} d x d y
$$

where $D$ is the region to be removed, that is to say, $I(x, y)$ is supposed to be unknown in $D$. The Euler-Lagrange solution to this optimization problem is the Laplace equation:

$$
\nabla^{2} I=I_{x x}+I_{y y}=0
$$

with the Dirichlet boundary condition $\left.I\right|_{\partial \bar{D}}(x, y)$, the restriction of the original image on the boundary of $D$ 's complement $\bar{D}=\Omega-D$ where $\Omega$ is the domain of definition of the entire image. Equation (8) defines a 2-dimensional harmonic function on the region to be removed, and thus we call this method "harmonic cut".

The Laplace equation is a special case of Poisson equation, which has been studied extensively [13]. In the actual implementation, a small scale is selected and elliptic features are detected. These features correspond to either noise or tiny substructures (approximately 20 pixels) on the nuclei. The corresponding regions are subsequently interpolated with a harmonic function. Figure 5(b) shows detected elliptic features at scale $\sigma=2$, where these bright dots, inside nuclei, are known to be chromatin. 


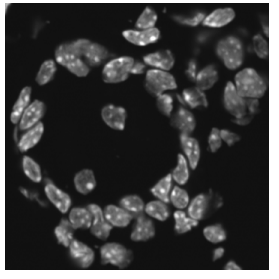

(a)

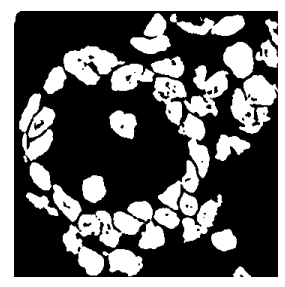

(d)

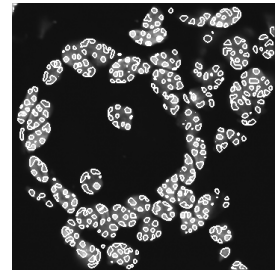

(b)

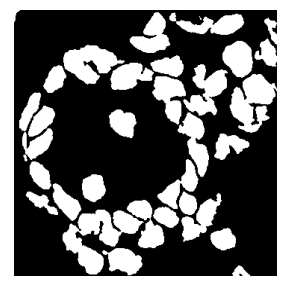

(e)

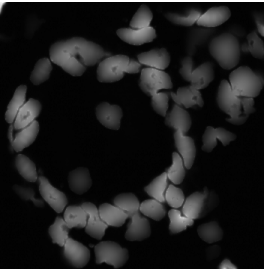

(c)

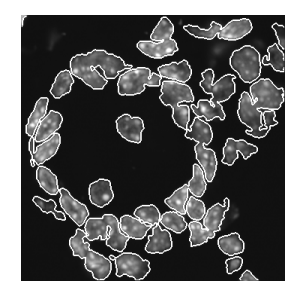

(f)
Fig. 5. Detection of elliptic features and their interpolation with harmonic cuts: (a) original image (same as Figure 1(a); (b) edges of bright elliptic features at scale $\sigma=2$; (c) harmonic cut on features in (b); (d) zero-crossings of (c); (e) removing small holes in (d); (f) displaying edge of (e) on the original image.

Figure 5 shows the result of the harmonic cut. It is clear that the edge detection has been improved when compared to Figure 1. The harmonic cut, shown in Fig.5(f), can deal with the failed cases in Figure 1.

\section{A. Properties of harmonic cut}

Harmonic functions are those functions that satisfy the Laplace equation. The following propositions can be found in any standard textbook [14] on complex/harmonic analysis.

Proposition 1: For any harmonic function, its value at any point is equal to the average of its values along any circle centered at that point, provided the function is defined within the circle.

Proposition 2: Any non-constant harmonic function has no maximum or minimum value inside the region in which it is defined.

Proposition 1 indicates that harmonic functions are smooth. In fact, Equation (7) shows that the maximum smoothness has been reached by harmonic functions. According to Proposition 2 , if we remove one region and replace it with the harmonic function defined by its boundary, there is no local minimum/maximum in the removed region. Hence, harmonic cut can remove all local singularities.

Koenderink introduced the concept of causality [12], [15], which means that new level surfaces must not be created in the scale-space representation when the scale parameter is increased. This is one of the most important requirements for any multiscale representation. The above analysis indicates that harmonic cut are even stronger because of the inherent properties of harmonic functions.

\section{B. Discussion and comparison}

This section concludes with a few insights into harmonic cut and comparison to previous research.

1) The algorithm can also be applied to the detection of dark regions, inside of nuclei, defined by $I_{x x}+I_{y y}>0$ and $I_{x x} I_{y y}-I_{x y}^{2}>0$, but ambiguities due to background may arise. However, once large bright regions are detected, they provide the context to detect small dark sub-regions.

2) Harmonic cut is used to interpolate a region that has been detected by elliptic features regardless of its intensity against immediate background.

3) Harmonic is applied for several iterations since the scale of elliptic feature detection is very conservative, and the detected features are usually smaller than their actual sizes. It can be shown that this process does converge by observing that the energy $\frac{1}{2} \iint I_{x}^{2}+I_{y}^{2} d x d y$ is always decreasing.

4) Other interpolation strategies, such as bilinear or nearestpoint interpolations, can be used to initialize the solution of the Laplace equation. However, smoothness in the bounded region is not guaranteed.

An alternative technique proposed by Yanowitz and Bruckstein [16] combines thresholding based on magnitude of the gradient followed by interpolating these points with the Laplacian equation. In contrast, our interpolation method applies to closed regions that are detected with elliptic features. Although Yanowitz and Bruckstein's method gives satisfactory results in many cases, their method fails in our data set due to the multiscale nature of cellular compartments.

\section{Regularized Centroid Transform}

At this stage of the computational process, each cell is represented with a smooth surface corresponding to each of its subcompartments. The next step of the process is to separate objects that are grouped together into a clump, e.g., touching one another. This is achieved using Regularized Centroid Transform (RCT), as shown in Figure 6. The intent is to map vectors originating from the boundary of an ellipse to its centroid. If these vectors can be computed, then the entire boundary can be grouped together. This is true for both boundaries and their interior points, e.g., grouping utilizes not only the edges but also the region information. The main issue is that the position of the critical centroid is unknown because the lines normal to the boundary of the ellipse do not intersect at a focal point.

\section{A. Local centroids}

Let $I(x, y)$ be the original intensity image. At each point $\left(x_{0}, y_{0}\right)$, its equal-height contour is defined by $I(x, y)=$ $I\left(x_{0}, y_{0}\right)$. Expanding and truncating the above equation using Taylor's series, we have the following estimation:

$$
I_{x} u+I_{y} v+\frac{1}{2}\left[I_{x x} u^{2}+2 I_{x y} u v+I_{y y} v^{2}\right]=0
$$

where $u=x-x_{0}$ and $v=y-y_{0}$, or in the standard form

$$
\frac{1}{2} w^{T} H w+b^{T} w=0
$$




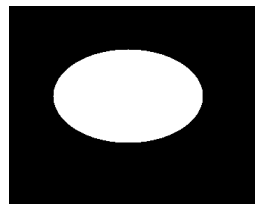

(a)

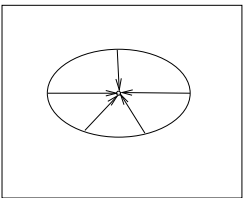

(b)
Fig. 6. Even though normal lines to the boundary of the ellipse do not intersect at a single point, RCT groups boundary points into a single focal point.

where $H=\left(\begin{array}{ll}I_{x x} & I_{x y} \\ I_{x y} & I_{y y}\end{array}\right)_{\left(x_{0}, y_{0}\right)}$ is the Hessian matrix, $b=$ $\left(\begin{array}{c}I_{x} \\ I_{y}\end{array}\right)_{\left(x_{0}, y_{0}\right)}$ is the gradient of intensity, $w=(u, v)^{T}$ is the centroid in the local coordinate system. Recall that the centroid of a quadratic curve, defined by Equation (10), satisfies the following linear constraint:

$$
H w+b=0
$$

If $H$ is non-singular, then the centroid can be determined directly, e.g. $w=-H^{-1} b$. However, this is not always true. If $H$ is singular and $b \neq 0$, there exists no solution.

The zero set defined by

$$
\left|\begin{array}{ll}
I_{x x} & I_{x y} \\
I_{x y} & I_{y y}
\end{array}\right|=I_{x x} I_{y y}-I_{x y}^{2}=0
$$

is non-trivial, and can be further classified into two categories:

1) uniform regions that correspond to zeros of intensity gradient of the image with the result that there is no information to estimate the centroid, and

2) elliptic features that occur in non-uniform regions.

\section{B. Regularized representation}

Computation of the local centroid is hindered due to the singularities of the Hessian and noise. The problem is inherently ill-posed and needs to be regularized [17]. Let the centroid at $(x, y)$ be denoted by $(u(x, y), v(x, y))^{T}$, then the regularized model can be expressed as:

$$
\begin{aligned}
& \min E(u, v)= \frac{1}{2} \iint\left\|H \cdot(u, v)^{T}+b\right\|^{2}+ \\
& \alpha\left(\|\nabla u\|^{2}+\|\nabla v\|^{2}\right) d x d y
\end{aligned}
$$

or

$$
\begin{aligned}
\min E(u, v)= & \frac{1}{2} \iint\left(I_{x x} u+I_{x y} v+I_{x}\right)^{2}+ \\
& \left(I_{x y} u+I_{y y} v+I_{y}\right)^{2}+ \\
& \alpha\left(u_{x}^{2}+u_{y}^{2}+v_{x}^{2}+v_{y}^{2}\right) d x d y
\end{aligned}
$$

where the first and second terms are the error of estimation, the third term is the smoothness constraint, and $\alpha(>0)$ is the weight factor.

Definition 2: We refer to the solution of Equation (14), a two-component vector field $\left(u^{*}, v^{*}\right):=\arg \min _{(u, v)} E(u, v)$, as the regularized centroid transform (RCT).

The main features of RCT are:

- RCT is a vector field that represents the displacement to the centroid at each point. This vector field is different from the normal vector. In the ideal case, shown by Figure 6 , RCT of every pixel on the boundary points to the same centroid, e.g., this vector field is well focused.

- The vector field is dense everywhere (even for binary image, a dense vector field is computed at each point).

- RCT is regularized under smoothness constraint. Smoothing is required since random and speckle noise can alter the position of the centroid.

- This is an intermediate step toward final segmentation.

\section{Discrete solution of RCT}

The Euler-Lagrange equations [18] of the variational problem Equation 14 are:

$$
\left\{\begin{array}{l}
I_{x x}\left(I_{x x} u+I_{x y} v+I_{x}\right)+I_{x y}\left(I_{x y} u+I_{y y} v+I_{y}\right)- \\
\alpha\left(u_{x x}+u_{y y}\right)=0 \\
I_{x y}\left(I_{x x} u+I_{x y} v+I_{x}\right)+I_{y y}\left(I_{x y} u+I_{y y} v+I_{y}\right)- \\
\alpha\left(v_{x x}+v_{y y}\right)=0
\end{array}\right.
$$

Substituting the finite difference approximations of the partial derivatives into the above partial differential equations, we have

$$
\left\{\begin{array}{l}
I_{x x}\left[I_{x x} u(x, y)+I_{x y} v(x, y)+I_{x}\right]+ \\
I_{x y}\left[I_{x y} u(x, y)+I_{y y} v(x, y)+I_{y}\right]- \\
\alpha[u(x+1, y)+u(x-1, y)+ \\
u(x, y+1)+u(x, y-1)-4 u(x, y)]=0 \\
I_{x y}\left[I_{x x} u(x, y)+I_{x y} v(x, y)+I_{x}\right]+ \\
I_{y y}\left[I_{x y} u(x, y)+I_{y y} v(x, y)+I_{y}\right]- \\
\alpha[v(x+1, y)+v(x-1, y)+ \\
v(x, y+1)+v(x, y-1)-4 v(x, y)]=0
\end{array}\right.
$$

which can be rewritten as follows

$$
\left\{\begin{array}{l}
a \cdot u(x, y)+b \cdot v(x, y)=e \\
c \cdot u(x, y)+d \cdot v(x, y)=f
\end{array}\right.
$$

where

$$
\begin{aligned}
a= & I_{x x}^{2}+I_{x y}^{2}+4 \alpha \\
b= & I_{x x} I_{x y}+I_{x y} I_{y y} \\
c= & b \\
d= & I_{x y}^{2}+I_{y y}^{2}+4 \alpha \\
e= & -I_{x} I_{x x}-I_{y} I_{x y}+\alpha[u(x+1, y)+u(x-1, y)+ \\
& u(x, y+1)+u(x, y-1)] \\
f= & -I_{x} I_{x y}-I_{y} I_{y y}+\alpha[v(x+1, y)+v(x-1, y)+ \\
& v(x, y+1)+v(x, y-1)]
\end{aligned}
$$

These coefficients are the functions of partial derivatives in the neighborhood of $(u(x, y), v(x, y))$. It is easy to show that the determinant $\Delta=a d-b c>16 \alpha^{2}$ is always positive, and the solution of Equation 17 is:

$$
\left\{\begin{array}{l}
u(x, y)=\frac{d e-b f}{\Delta} \\
v(x, y)=\frac{-c e+a f}{\Delta}
\end{array}\right.
$$

Hence we can compute a new set of estimates $\left(u^{n+1}, v^{n+1}\right)$ from the estimated partial derivatives and the previous estimates $\left(u^{n}, v^{n}\right)$ by

$$
\left\{\begin{array}{l}
u^{n+1}(x, y)=\frac{d e^{n}-b f^{n}}{\Delta} \\
v^{n+1}(x, y)=\frac{-c e^{n}+a f^{n}}{\Delta}
\end{array}\right.
$$


The initial solution $\left(u^{0}, v^{0}\right)$ can be set to 0 . The boundary condition for solution of the above PDEs are based on Neumann boundary conditions: $\left\{\begin{array}{l}\frac{\partial u}{\partial \mathbf{n}}=0 \\ \frac{\partial v}{\partial \mathbf{n}}=0\end{array}\right.$ where $\mathbf{n}$ is the normal vector to the boundary. For the Neumann boundary condition, it is assumed that the data outside the domain of definition are a reflection of the data inside the domain.

\section{Speed-up}

The representation just described indicates that the computational complexity of RCT is very high. However, a number of coefficients can be precomputed. Let's rewrite $e$ and $f$ in the following form:

$$
\begin{aligned}
& e=e_{0}+4 \alpha \bar{u} \\
& f=f_{0}+4 \alpha \bar{v}
\end{aligned}
$$

where

$$
\begin{aligned}
\epsilon_{0} & =-I_{x} I_{x x}-I_{y} I_{x y} \\
\bar{u} & =\frac{1}{4}[u(x+1, y)+u(x-1, y)+u(x, y+1)+u(x, y-1)] \\
f_{0} & =-I_{x} I_{x y}-I_{y} I_{y y} \\
\bar{v} & =\frac{1}{4}[v(x+1, y)+v(x-1, y)+v(x, y+1)+v(x, y-1)]
\end{aligned}
$$

Then Equation 20 can be rewritten as

$$
\left\{\begin{array}{l}
u^{n+1}(x, y)=\frac{d\left(e_{0}+4 \alpha \bar{u}^{n}\right)-b\left(f_{0}+4 \alpha \bar{v}^{n}\right)}{\Delta} \\
v^{n+1}(x, y)=\frac{-c\left(e_{0}+4 \alpha \bar{u}^{4}\right)+a\left(f_{0}+4 \alpha \bar{v}^{n}\right)}{\Delta}
\end{array}\right.
$$

or

$$
\left\{\begin{array}{l}
u^{n+1}=u_{0}+\kappa_{11} \bar{u}^{n}+\kappa_{12} \bar{v}^{n} \\
v^{n+1}=v_{0}+\kappa_{21} \bar{u}^{n}+\kappa_{22} \bar{v}^{n}
\end{array}\right.
$$

where

$$
\left\{\begin{array}{l}
u_{0}=\frac{d e_{0}-b f_{0}}{\Delta} \\
v_{0}=\frac{-c e_{0}+a f_{0}}{\Delta} \\
\kappa_{11}=\frac{4 \alpha d}{\Delta} \\
\kappa_{12}=-\frac{4 \alpha b}{\Delta} \\
\kappa_{21}=-\frac{4 \alpha c}{\Delta} \\
\kappa_{22}=\frac{4 \alpha a}{\Delta}
\end{array}\right.
$$

are coefficients independent of the iteration time. They can be precomputed and stored. As a result, each iteration needs computation of 4-point averages of $u$ and $v$. It is easy to show that its computational complexity is of the same order as the linear diffusion.

\section{PARTITIONING VECTOR FIELD}

The final step of segmentation is to compute a partition for RCT. Consider an autonomous system of differential equations

$$
\left\{\begin{aligned}
\frac{d x}{d t} & =u(x, y) \\
\frac{d y}{d t} & =v(x, y)
\end{aligned}\right.
$$

Definition 3: An orbit or trajectory of the system (26) is a set $\omega \subset \Omega$ of the form $\{(x(t), y(t)) \mid t \in T\}$, where $(x(t), y(t))$ is a solution of (26) defined on time interval $T$.

Definition 4: An equilibrium point of the system (26) (also called a critical or singular point) is a point $\left(x^{0}, y^{0}\right) \in \Omega$ such that $u\left(x^{0}, y^{0}\right)=0$ and $v\left(x^{0}, y^{0}\right)=0$.

Theorem 1: (a) Every point of $\Omega$ belongs to precisely one orbit of the system (26). (b) If $\left(x^{0}, y^{0}\right)$ is an equilibrium point of the system then $\left\{\left(x^{0}, y^{0}\right)\right\}$ is an orbit.

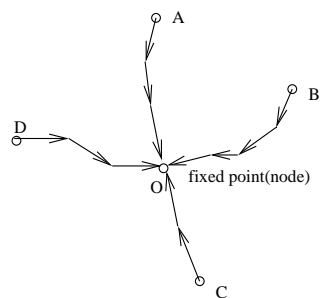

Fig. 7. Partition of vector field: points A, B, C, and D are grouped into one region.

According to this theorem, a partition RCT can be obtained as follows. Every non-singular pixel can be repeatedly moved to a new position by solving Equation (26), and every singular point is in fact a fixed (possibly unstable) point. Those points that move to the same singular point can be grouped together. This grouping technique is illustrated in Figure 7. There may be some exceptions in this strategy: (1) when a singular point may go out of the image domain, and (2) both eigenvalues of a singular point are purely imaginary; (3) there may be limit cycles. In these cases, we simply discard these points. Since we have not found limit cycles in any of our experiments, the conjecture is that there is no limit cycle in RCT.

\section{EXPERIMENTAL RESULTS AND COMPARISON}

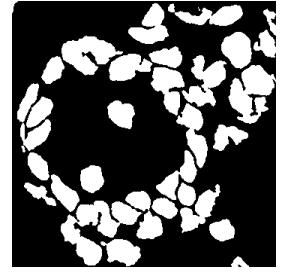

(a)

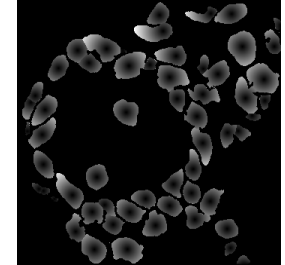

(b)

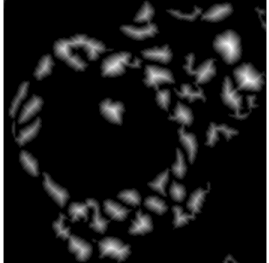

(c)
Fig. 8. Comparison of RCT and distance transform: (a) Original binary image; (b) RCT energy function shows a smooth surface and a fixed point for each region; (c) traditional distance transform has many local maxima.

We have implemented Vincent and Soille's watershed algorithm [19] for comparative analysis. Their method works well only when the boundaries are smooth. Yet, the boundary is often noisy, which leads to oversegmentation. Figure 8(b)(c) compare the RCT energy function (the arclength of the trajectory) with the traditional distance transform. It is clear that RCT is smooth and each region has a corresponding fixed point. Figures 9 shows segmentation results for two fields of cells. and the comparison to the watershed technique, which indicates significant oversegmentation. We have applied our technique to 68 images with a total of 2417 cells, and 61 cells were incorrectly segmented. Some of these images can be found in http://vision.lbl.gov/Projects/BioSig in the "Image" category. 


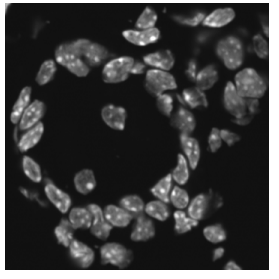

(a)

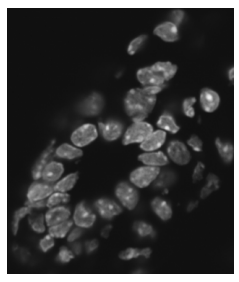

(d)

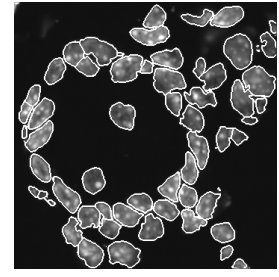

(b)

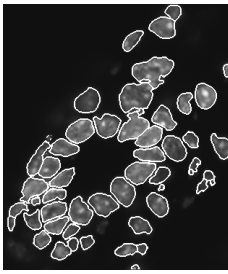

(e)

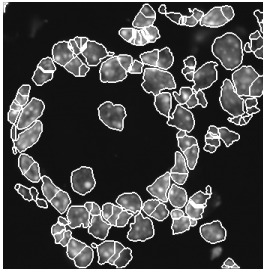

(c)

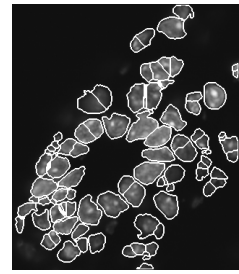

(f)
Fig. 9. Segmentation of two fields of cells: (column 1) the original images; (column 2) results by our approach (harmonic cut + RCT); (column 3) oversegmented results by the "distance transform + watershed" strategy.

\section{CONCLUSION}

This paper has outlined a layered computational technique to delineate overlapping nuclei and to extract their internal substructures. Our approach is model-based and we have developed computational operators to eliminate the need for ad-hoc techniques. The first step of the process is removal of random and speckled (internal substructure) noise, which are subsequently interpolated with harmonic cut. The next step of the process delineates overlapping regions through a novel process that we call regularized centroid transform (RCT). In each step of the process, we have given examples and compared our results to previous research. Our approach has been applied to high throughput analysis of images obtained through a confocal microscope, and it is currently in production use. It is possible for the proposed method to produce incorrect segmentation due to inherent ambiguities that are present from a single focal plane, e.g., 2D analysis. A more robust approach will need $3 \mathrm{D}$ analysis. We suggest that RCT is a generic blob detection and separation technique that can be used as a generic tool for early vision problems.

\section{ACKNOWLEDGMENT}

Research was funded by the Medical Sciences Division and Mathematical, Information, and Computing Sciences Division, of the U. S. Department of Energy under Contract No. DE-AC03-76SF00098 with the University of California. The publication number is LBNL-51849.

\section{REFERENCES}

[1] A. Garrido and N. de la Blanca, "Applying deformable templates for cell image segmentation," $P R$, vol. 33, no. 5, pp. 821-832, May 2000.
[2] $\mathrm{H}$. Wu, J. Barba, and J. Gil, "Iterative thresholding for segmentation of cells from noisy images," Journal of Microscopy, vol. 197(3), pp. 296-304, 2000.

[3] K. Wu, D. Gauthier, and M. Levine, "Live cell image segmentation," IEEE Trans. on Biomedical Engineering, vol. 42(1), pp. 1-12, 1995.

[4] B. Parvin, Q. Yang, G. Fonteny, and M. Barcellos-Hoff, "Biosig: An imaging bioinformatic system for studying phenomics," IEEE Computer, vol. 35, pp. 65-71, 2002.

[5] B. Loo, B. Parvin, and S. Rothman, "Two- and three- dimensional measurement of particles in the analysis of microscopic digital images of biological samples," in The International Society for Optical Engineering, 1996, pp. 49-52.

[6] G. Cong and B. Parvin, "Model-based segmentation of nuclei," Pattern Recognition, vol. 33, no. 8, pp. 1383-1393, 2000.

[7] A. Sarti, C. Solorzano, S. Lockett, and R. Malladi, "A geometric model for 3-d confocal image analysis," IEEE Trans. on Biomedical Engineering, vol. 47(12), pp. 1600-1609, 2000.

[8] S. Sclaroff and L. Liu, "Deformable shape detection and description via model-based region grouping," PAMI, vol. 23, no. 5, pp. 475-489, May 2001.

[9] Y. Jin and R. Sinniah, "Clump splitting through concavity analysis," Journal of Pattern Recognition, vol. 15, pp. 1013-1018, 1994.

[10] A. Nedzved, S. Ablameyko, and I. Pitas, "Morphological segmentation of histology cell images," in ICPRO0, 2000, pp. Vol I: 500-503.

[11] W. Wang, "Binary image segmentation of aggregates based on polygonal approximation and classification of concavities," Journal of Pattern Recognition, vol. 31, pp. 1502-1524, 1998.

[12] T. Lindeberg, "Scale-space theory: A basic tool for analyzing structures at different scales," Journal of Applied Statistics, pp. 225-270, 1994.

[13] T. Simchony, R. Chellappa, and M. Shao, "Direct analytical methods for solving poisson equations in computer vision problems," PAMI, vol. 12 , no. 5, pp. 435-446, May 1990.

[14] L. Alfors, Complex Analysis. McGraw-Hill, 1966.

[15] J. Koenderink, "The structure of images," Biol. Cybern., vol. 50, pp. 363-370, 1995.

[16] S. Yanowitz and A. Bruckstein, "A new method for image segmentation," CVGIP, vol. 46, no. 1, pp. 82-95, April 1989.

[17] A. Tikhonov, "The regularization of ill-posed problems," Dokl. Akad. Nauk., vol. SSR 153, no. 1, pp. 49-52, 1963.

[18] E. Zeidler and L. Boron, Nonlinear Functional Analysis and Its Applications, Part 3: Variational Methods and Optimization. Springer-Verlag, 1984.

[19] L. Vincent and P. Soille, "Watersheds in digital spaces: An efficient algorithm based on immersion simulations," PAMI, vol. 13, no. 6, pp. 583-598, June 1991.

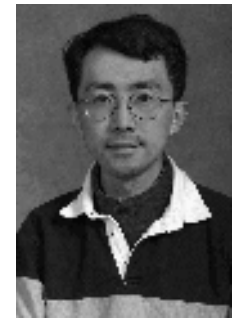

Qing Yang is a computer scientist in the Computational Science Division at Lawrence Berkeley National Laboratory. His research interests include image processing, pattern recognition and bioinformatics. He received a $\mathrm{PhD}$ in computer science from the Institute of Automation, Chinese Academy of Sciences. Contact him at: qyang@media.lbl.gov.

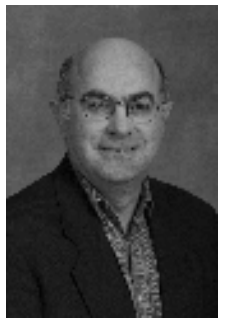

Bahram Parvin is a staff scientist in the Computing Sciences of Lawrence Berkeley National Laboratory. $\mathrm{He}$ conducts research on bio-imaging, feature-based representation of time varying scientific images, and distributed informatics systems. He received his Ph.D. in Electrical Engineering from the University of Southern California in 1991. He is a senior member of IEEE. 